\title{
The Ionization of the Warm Gas in Early-type Galaxies and Its UV Upturn
}

\author{
Renbin Yan $^{1,2}$ and Michael R. Blanton ${ }^{2}$ \\ ${ }^{1}$ Department of Physics and Astronomy, University of Kentucky, Lexington, KY, 40506, USA \\ email: renbin@pa.uky.edu \\ ${ }^{2}$ Center for Cosmology and Particle Physics, Department of Physics, New York University, \\ New York, NY, 10003, USA \\ email: michael.blanton@nyu.edu
}

It has long been known that the majority of early-type galaxies contain warm ionized gas producing optical emission lines. These warm ionized gas are spatially extended to kpc scales. Their line ratios satisify the criteria of Low-ionization Nuclear Emission-line Regions (LINERs) on all major line-ratio diagnostic diagrams. However, their ionization mechanism has been hotly debated. Multiple ionization mechanisms can produce the same kind of line ratios, including photoionization by a central AGN, photoionization by hot evolved stars (e.g. post-AGB stars), collisional ionization by fast shocks, photoionization by hot X-ray emitting gas, and conductive heating or turbulent mixing. Therefore, determining the ionization mechanism requires other information.

Measuring the spatial gradient in a line ratio that is sensitive to ionization parameter will allow us to distinguish a central ionizing source from distributed ionizing sources. We utilized the SDSS survey data to measure this. SDSS used a fixed angular aperture of 3", which corresponds to different physical scales at different distances. By selecting the same population of galaxies at all distances, we can statistically study the spatial distribution of line emission and line ratio gradient. We found that the median line ratio gradient in $[\mathrm{O}$ III $] /[\mathrm{S} \mathrm{II}]$ among non-star-forming red galaxies changes very slowly with increasing physical scale covered, which rules out AGN as the ionizing source and strongly favors a distributed ionizing sources that follow the stellar density profile. Additionally, the stellar photoionizing model predicts that the line ratio gradient is dependent on luminosity. Since the stellar density profile is different between bright and faint early-type galaxies, they should produce different ionizing flux profiles and different line ratio gradients. By separting the sample accoring to broadband luminosity, we verified this prediction using the SDSS data. This strongly suggests that the ionizing source is spatially distributed like the stars. For details, see Yan \& Blanton (2012).

To evaluate the likelihood of the shock ionization and turbulent mixing models, we measured the temperature of the gas in coadded spectra. We selected non-star-forming red galaxies in SDSS and separated them into three gas-metallicity bins according to $[\mathrm{N} \mathrm{II}] /[\mathrm{O} \mathrm{II}]$ ratio. After carefully removing the stellar continuum, we were able to measure the weak [N II] $\lambda 5755$ line. The [N II] 6584/5755 ratio yields a temperature measurement of $15,000 \mathrm{~K}$ for the low-metallicity sample and $8,000 \mathrm{~K}$ for the high-metallicity sample. These temperatures are consistent with the photoionization but are inconsistent with shock models or turbulent mixing models.

Therefore, we conclude that the warm ionized gas in the majority of early-type galaxies are most likely ionized by the UV-bright old stellar population.

\section{Reference}

Yan, Renbin, Blanton, \& Michael R., 2012 Astrophys. J., 747, 61 